\title{
Correspondence
}

Psychological Medicine, 46 (2016). doi:10.1017/S003329171600009X

First published online 18 February 2016

\section{Letter to the Editor \\ Should we focus on quality or quantity in meta-analyses?}

In an invited commentary (Moritz et al. 2016), the developers of metacognitive training (MCT) have challenged the results of the meta-analysis on the efficacy of MCT on positive symptoms, delusions and data gathering bias (van Oosterhout et al. 2015). The recommendation of this meta-analysis was not to implement $\mathrm{MCT}$ in routine care at this moment, because of a lack of evidence.

In their introduction (Moritz et al. 2011) refer to another meta-analysis by Jiang et al. (2015) quoting significant effects for positive symptoms (as well as for delusions without the study by van Oosterhout et al. 2014). The van Oosterhout et al. (2014) study is a negative study, but it is the largest randomized controlled trial (RCT) and indeed was rated as the study with the lowest risk of bias by Jiang et al.; it is not clear why one would discard the best study to date? The developers could usefully be referred to the conclusion in the abstract of Jiang et al:: 'The limited number of RCT trials, the variability of the method and time of the outcome evaluation, and methodological problems in the trials make it impossible to come to a conclusion about the effectiveness of MCT for schizophrenia. More randomized trials that use standardized outcome measures, that use intention-to-treat (ITT) analyses, and that follow-up participants at regular intervals after the intervention are needed to determine whether or not MCT should become a recommended adjunctive treatment for schizophrenia.' In our opinion this conclusion is the same as ours.

In their rebuttal the developers apply four headings with different arguments; these are addressed below.

\section{Studies omitted}

Moritz et al. note that some studies were omitted; we reran our analyses including the appropriate studies. We added the data of Aghotor et al. (2010); Moritz et al. (2011) and Gaweda et al. (2015). We found the data in the meta-analysis of Eichner (2015), a PhD student of Moritz, who was kind enough to provide the data via Research Gate. We discarded two nonrandomized studies: an extremely negative study by
Rocha \& Queirós (2013) and an extremely positive study by Erawati et al. (2014).

It is not accurate to say that we omitted the So et al. (2015) study from our paper because it had not been published - or accepted for publication - at the point our meta-analysis was accepted for publication.

However, we have added this study in our reanalysis.

The results of the reanalysis demonstrate that there are some significant effects for positive symptoms $(g=0.32)$ and delusions $(g=0.31)$, but not for data gathering $(g=$ 0.11) if all the studies are considered. However, the findings from the high-quality and intention-to-treat data are similar to our original results with no significant effects on delusions, positive symptoms or data gathering.

\section{Heterogeneity and moderator analysis}

We chose to conduct some additional moderator analyses with the high-quality studies and not the low-quality studies as Moritz et al. suggest. Van Oosterhout et al. (2014) is the largest study with the lowest risk of bias, but is a negative study. It does not make sense to remove robust studies from an analysis only to remove heterogeneity.

\section{$\mathrm{MCT}+$ is not CBT}

The authors (Moritz et al. 2011b) wrote in the paper on $\mathrm{MCT}+$ that 'Individualized metacognitive therapy $(\mathrm{MCT}+)$ followed group sessions according to the general guidelines for CBT (e.g. Fowler et al. 1995).' As Fowler is one of the founding fathers of CBT for psychosis, this led us to conclude that MCT+ is based on CBT. In the same paper it is stated: 'MCT ... is grounded on the principles of CBT (Fowler et al. 1995) and basic research on cognitive biases in schizophrenia (for reviews, see Garety \& Freeman, 1999; Freeman et al. 2007), as well as deficits in social cognition/theory of mind (Frith, 1994; Frith \& Corcoran, 1996).' CBT in psychosis has included work on cognitive biases for over a decade. The main difference seems to be that MCT is set up like a course targeting transfer of 'cold cognitions', while CBT focuses on personalizing and experiencing the effects of biases in real life. In doing so, CBT is rather aimed at modifying 'hot cognitions'.

\section{Evolution of MCT}

Moritz and colleagues suggested changing the inclusion criteria to patients without severe delusions. We 
have checked the baseline data of the studies on delusions. The three studies with the highest baseline delusion scores were So et al. (2015), Favrod (2014) and van Oosterhout et al. (2014). Taking these three studies, the effect size is 0.49 , while studies with the lowest delusion scores at baseline (Moritz et al. 2011a,b, 2013; Briki et al. 2014; Gaweda et al. 2015) have a pooled effect size of 0.25 on delusions. So the empirical evidence rather suggests that patients with highest baseline scores of paranoia in general benefit most from therapy.

In summary, we appreciate the opportunity to air these differences in opinion; but have to conclude that the best available evidence suggests that MCT is not yet at a stage to advocate its routine use. That is to say, we acknowledge Moritz et al.'s position that MCT is work in progress and this progress needs to be data driven. However, including less rigorous evidence into a meta-analysis may offer a different opinion, but this is probably not the most robust scientific way forward.

\section{Declaration of Interest}

None.

\section{References}

Aghotor J, Pfueller U, Moritz S, Weisbrod M, Roesch-Ely D (2010). Metacognitive training for patients with schizophrenia (MCT): feasibility and preliminary evidence for its efficacy. Journal of Behavior Therapy and Experimental Psychiatry 41, 207-211.

Briki M, Monnin J, Haffen E, Sechter D, Favrod J, Netillard C, Vandel P (2014). Metacognitive training for schizophrenia: a multicentre randomised controlled trial. Schizophrenia Research 157, 99-106.

Eichner C (2015). Can metacognitive training (MCT) reduce positive symptoms and delusions in patients with schizophrenia? A meta-analysis taking into account important moderators. University of Hamburg: Hamburg.

Erawati E, Keliat BA, Helena N, Hamid A (2014). The influence of metacognitive training on delusion severity and metacognitive ability in schizophrenia. Journal of Psychiatric and Mental Health Nursing 21, 841-847.

Favrod J, Rexhaj S, Bardy S, Ferrari P, Hayoz C, Moritz S, Conus P, Bonsack C (2014). Sustained antipsychotic effect of metacognitive training in psychosis: a randomized controlled study. European Psychiatry 29, 275-281.

Fowler D, Garety P, Kuipers E (1995). Cognitive Behaviour Therapy for Psychosis: Theory and Practice. Wiley, Chichester, UK.

Gaweda L, Krezolek M, Olbrys J, Turska A, Kokoszka A (2015). Decreasing self-reported cognitive biases and increasing clinical insight through meta-cognitive training in patients with chronic schizophrenia. Journal of Behavior Therapy and Experimental Psychiatry 48, 98-104.

Jiang J, Zhang L, Zhu Z, Li W, Li C (2015). Metacognitive training for schizophrenia: a systematic review. Shanghai Archives of Psychiatry 27, 149-157.
Moritz S, Kerstan A, Veckenstedt R, Randjbar S, Vitzthum F, Schmidt C, Woodward TS (2011a). Further evidence for the efficacy of a metacognitive group training in schizophrenia. Behaviour Research and Therapy 49, 151-157.

Moritz S, Veckenstedt R, Randjbar S, Vitzthum F, Woodward TS (2011b). Antipsychotic treatment beyond antipsychotics: metacognitive intervention for schizophrenia patients improves delusional symptoms. Psychological Medicine 41, 1823-1832.

Moritz S, Veckenstedt R, Bohn F, Hottenrott B, Scheu F, Randjbar S, Aghotor J, Kother U, Woodward TS, Treszl A, Andreou C, Pfueller U, Roesch-Ely D (2013).

Complementary group metacognitive training (MCT) reduces delusional ideation in schizophrenia. Schizophrenia Research 151, 61-69.

Moritz S, Werner D, Menon M, Balzan RP, Woodward TS (2016). Jumping to negative conclusions - a case of studygathering bias? Psychological Medicine 46, 59-61.

Rocha NB, Queirós C (2013). Metacognitive and social cognition training (MSCT) in schizophrenia: a preliminary efficacy study. Schizophrenia Research 150, 64-68.

So SH, Chan AP, Chong CS, Wong MH, Lo WT, Chung DW, Chan SS (2015). Metacognitive training for delusions (MCTd): effectiveness on data-gathering and belief flexibility in a Chinese sample. Frontiers in Psychology 6, 730.

van Oosterhout B, Krabbendam L, de Boer K, Ferwerda J, van der Helm M, Stant AD, van der Gaag M (2014). Metacognitive group training for schizophrenia spectrum patents with delusions: a randomized controlled trial. Psychological Medicine 44, 3025-3035.

van Oosterhout B, Smit F, Krabbendam L, Castelein S, Staring AB, van der Gaag M (2015). Metacognitive training for schizophrenia spectrum patients: a meta-analysis on outcome studies. Psychological Medicine. Published online: 20 July 2015. doi:10.1017/s0033291715001105

B. VAN OOSTERHOUT ${ }^{1,}{ }^{*}$, F. SMIT ${ }^{2,3,4}$, L. KRABBENDAM ${ }^{5}$, S. CASTELEIN ${ }^{6,7}$, A. B. P. STARING ${ }^{8}$ AND M. VAN DER $\mathrm{GAAG}^{4,9}$

${ }^{1}$ GGzE, De Woenselse Poort, Eindhoven, The Netherlands

${ }^{2}$ Trimbos Institute (Netherlands Institute of Mental Health and Addiction), Utrecht, The Netherlands

${ }^{3}$ Department of Epidemiology and Biostatistics, EMGO Institute for Health and Care Research, VU University Medical Centre, Amsterdam, The Netherlands

${ }^{4}$ Department of Clinical Psychology, EMGO Institute for Health and Care Research, VU University, Amsterdam, The Netherlands

${ }^{5}$ Department of Educational Neuroscience and Research Institute Learn!,Faculty of Psychology and Education, VU University, Amsterdam, The Netherlands

${ }^{6}$ Lentis Psychiatric Institute, Lentis Research, Groningen, The Netherlands

${ }^{7}$ University of Groningen, University Medical Center Groningen, Rob Giel Research Center, Groningen, The Netherlands 
${ }^{8}$ Altrecht Psychiatric Institute, Utrecht, The Netherlands ${ }^{9}$ Department of Psychosis Research, Parnassia Psychiatric Institute, The Hague, The Netherlands
*Address for correspondence: B. van Oosterhout, GGzE, PO Box 909, 5600 AX, Eindhoven, The Netherlands.

(Email: bj.van.oosterhout@dewoenselsepoort.nl) 